\title{
Installation and operation of the LHCb Silicon Tracker detector
}

D. Esperante ${ }^{*, a}$, A. Bay ${ }^{\mathrm{b}}$, F. Blanc ${ }^{\mathrm{b}}$, M.O. Bettler ${ }^{\mathrm{b}}$, G. Conti ${ }^{\mathrm{b}}$, V. Fave ${ }^{\mathrm{b}}$, R. Frei ${ }^{\mathrm{b}}$, N. Gueissaz ${ }^{\mathrm{b}}$, G. Haefeli ${ }^{\mathrm{b}}$, A. Keune $^{\mathrm{b}}$, J. Luisier ${ }^{\mathrm{b}}$, R. Muresan ${ }^{\mathrm{b}}$, T. Nakadab ${ }^{\mathrm{b}}$, M. Needham ${ }^{\mathrm{b}}$, L. Nicolas ${ }^{\mathrm{b}}$, M. Knecht $^{\mathrm{b}}$, A. Perrin ${ }^{\mathrm{b}}$, C. Potterat ${ }^{\mathrm{b}}$, O.

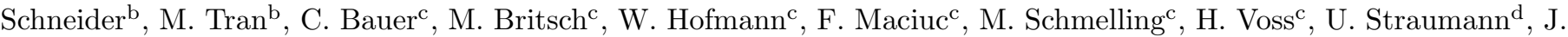
Anderson $^{d}$, A. Buechler ${ }^{d}$, N. Chiapolini ${ }^{d}$, V. Hangartner ${ }^{d}$, C. Salzmann ${ }^{d}$, S. Steiner ${ }^{d}$, O. Steinkamp ${ }^{d}$, J. van Tilburg ${ }^{d}$, A. Vollhardt ${ }^{\mathrm{d}}$, B. Adeva ${ }^{\mathrm{a}}$, J. Fungueiriño Pazos $^{\mathrm{a}}$, A. Gallas ${ }^{\mathrm{a}}$, A. Pazos Alvarez ${ }^{\mathrm{a}}$, E. Pérez Trigo ${ }^{\mathrm{a}}$, M. Pló Casasús ${ }^{\mathrm{a}}$, P.

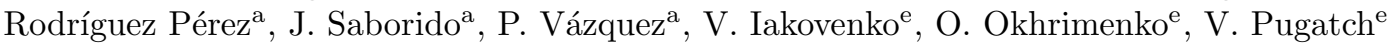

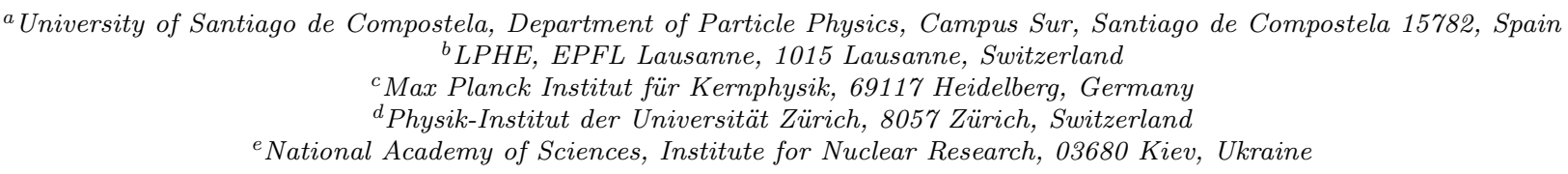

\begin{abstract}
The LHCb experiment is designed to perform high-precision measurements of CP violation and rare decays of B hadrons. The construction and installation of the Silicon Tracker (ST) was completed by early summer 2008. The Silicon Tracker covers a sensitive area of about $12 \mathrm{~m}^{2}$ using silicon micro-strip technology and must withstand charged particle fluxes of up to $5 \times 10^{5} \mathrm{~cm}^{-2} \mathrm{~s}^{-1}$. In these proceedings the preparation of the detectors for the first LHC beams is reported. Selected results from the detector commissioning in $\mathrm{LHCb}$ are shown, including the first beam-related events accumulated during the LHC injection and synchronization tests in September 2008. Lessons are drawn from the experience gathered during the installation and commissioning.
\end{abstract}

Key words: LHCb, Silicon, Tracker, Semiconductor Detectors PACS: 07.77.Ka, 29.40.Wk

\section{Introduction}

The Silicon Tracker is part of the tracking system of the $\mathrm{LHCb}$ experiment [1]. $\mathrm{LHCb}$ is a single-arm spectrometer with excellent tracking and particle identification capabilities. It covers an acceptance out to $250 \mathrm{mrad} \times 300$ mrad around the LHC beam axis. The experiment has been designed to perform high-precision measurements of CP violation and rare decays of B hadrons. The Silicon Tracker (ST) consists of two detectors, both of which use silicon micro-strip technology with long read-out strips, giving a total active area of $\sim 12 \mathrm{~m}^{2}$ with $\sim 272 \mathrm{k}$ read-out channels. The first of these detectors, the Tracker Turicensis (TT), is a $\sim 150 \mathrm{~cm}$ wide and $\sim 130 \mathrm{~cm}$ height planar tracking station that is placed upstream of the $\mathrm{LHCb} 4$ Tm dipole magnet and covers the whole acceptance of the experiment (Fig. 1). The second of the two detectors is the Inner Tracker (IT). It covers a $125 \mathrm{~cm}$ wide and $40 \mathrm{~cm}$ high cross-shaped region (Fig. 2) in the center of the three planar tracking stations downstream of the magnet (Fig. $1)$. Each of the four ST stations comprises four detection layers ( 2 with $\pm 5^{\circ}$ stereo angle). The same front-end radi- ation hard read-out chip (the Beetle [2]) and read-out link [3] are used both in the IT and the TT.
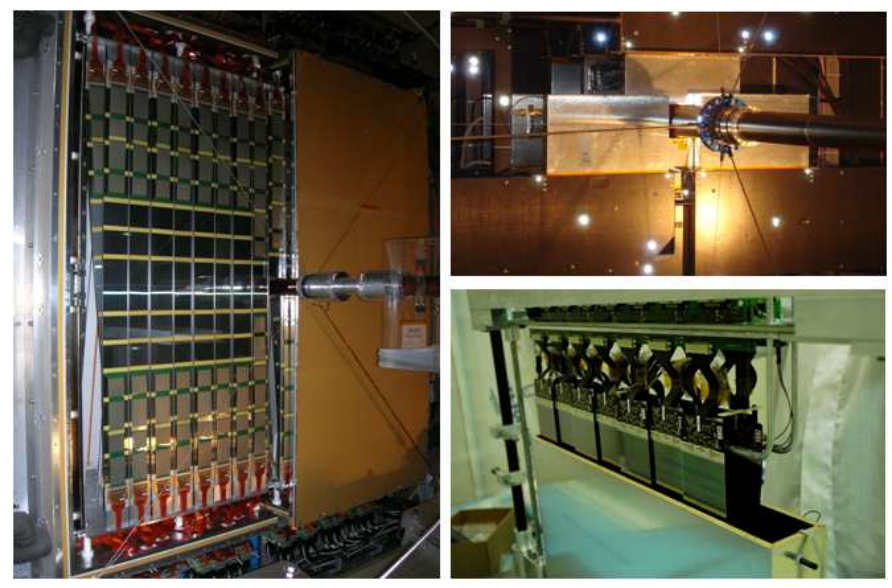

Figure 1: View of the TT station during the installation at $\mathrm{LHCb}$ (left). View of the first IT station installed at $\mathrm{LHCb}$ (top right), and detail of one of the detector boxes (bottom right).

\footnotetext{
*Corresponding author. Tel.: +34-981-563100-14012; fax: +34981-521091.

Email address: daniel.esperante@usc.es (D. Esperante)
} 


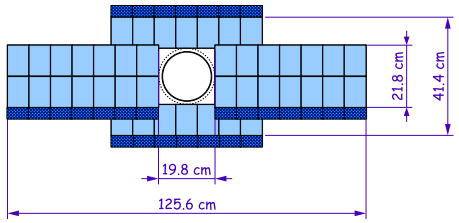

Figure 2: Station layout of an IT x-layer.

\section{Module design, production and quality testing}

The module design and production is described in detail in [4] and references therein. Up to 4 p-on-n siliconstrip sensors (HPK) are bonded together to give read-out strips up to $37.2 \mathrm{~cm}$ in length. The pitch is $183 \mu \mathrm{m}$ (TT) or $198 \mu \mathrm{m}$ (IT) with $\mathrm{w} / \mathrm{p}=0.25$ and the thicknesses are $320,410,500 \mu \mathrm{m}$ depending on the location of the module in the detector. The production of $\sim 400$ IT and $\sim 150$ TT modules was finished at the two production sites in $\mathrm{Au}-$ tumn 2007. Quality assurance played an important role at all stages of the module production. The silicon sensors were qualified at the factory and re-tested upon reception at the production sites confirming the good quality reported by the manufacturer. Tests against infant mortality were performed on the modules at various steps during the assembly; the tests comprised temperature cycling and electrical burn-in for up to $48 \mathrm{~h}$. Shorted and open channels were spotted using the internal Beetle test pulse by analyzing both the strip noise and pulsed signal level. Pedestal runs with the detector box opened allowed pinholes to be identified. The light shining onto the sensors generates leakage currents in the silicon bulk, which, in presence of a pinhole, saturate the front-end pre-amplifier. Metrology measurements were performed before and after assembly of the detector module. The positioning accuracy obtained is much better than the expected spatial resolution of about $50 \mu \mathrm{m}$.

\section{Commissioning and first data results}

The installation of the ST was complete by early summer 2008. At the end of September, the TT had more than $99 \%$ of working channels, for the IT $97 \%$ of the channels were fully functioning. The remaining problems have been identified and are being fixed at the time of writing. Commissioning with particles has started as well. We have used cosmic events, albeit at a low rate due to the horizontal geometry of $\mathrm{LHCb}$, to perform a coarse time alignment of the detector. From 2.55 million acquired cosmic triggers there are several hundred particles that pass through at least one of the IT boxes. Eighty six of these events contain track segments that cross neighboring stations and two events have a track that could be reconstructed in all three Inner Tracker stations. In Figure 3 one of the latter events is shown, in which ten out of the twelve possible hit layers are included in the reconstructed track. One hit is outside of the search window while the last one is missing. The unbiased hit residuals of the reconstructed track segments that cross at least two Inner Tracker stations are displayed in Figure 4. Reconstruction with the nominal detector geometry results in an RMS of $310 \mu \mathrm{m}$, by including the information from a partial survey this number is reduced to $270 \mu \mathrm{m}$. Finally using the relative alignment information from events taken during the LHC injection tests an RMS of $254 \mu \mathrm{m}$ can be achieved. These results are reasonable taking into account the tolerances of the survey and the positioning of the detector [5]. The module to module relative alignment is estimated to $50 \mu \mathrm{m}$ and the box positioning to $1 \mathrm{~mm}$.

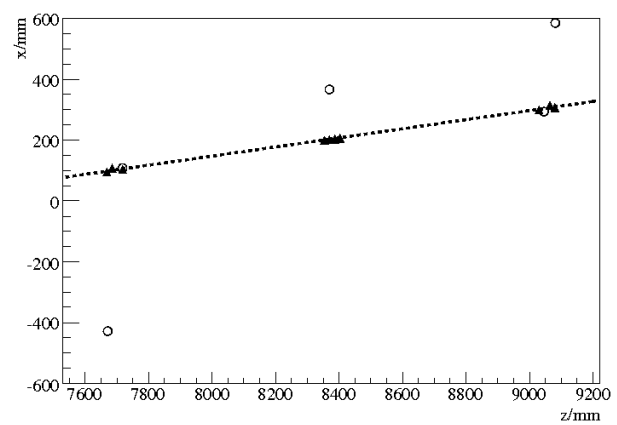

Figure 3: Reconstructed track of a cosmic particle in the Inner Tracker in $\mathrm{x}-\mathrm{z}$ projection with 10 hits. The circles are unassigned hits in the event.

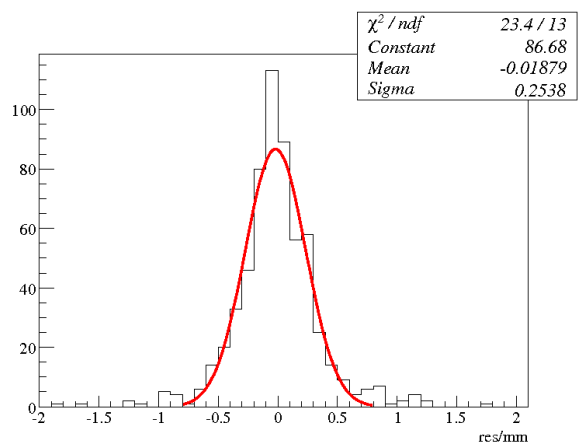

Figure 4: Unbiased residuals of reconstructed track segments from cosmic ray particles that cross at least two Inner Tracker station boxes.

Apart from the limited cosmic data, we successfully exploited the first LHC injection tests. In these tests the beam was dumped on to a beam stopper (the 'TED') in the SPS-LHC transfer line, 350 meters behind LHCb. During these tests the particle multiplicity was of about 20 times higher than that expected during normal running. The high multiplicity makes the reconstruction of individual tracks in the Silicon Tracker challenging. Nevertheless a simple algorithm has been used to provide a first alignment of the detector boxes and layers in $\mathrm{x}$. It consists of seeking track candidates in the expected direction from hits in the first and last layer and requiring a third hit to confirm 
the track. Hit residuals in middle box are then calculated. The residual distributions (see figure 5) are used to adjust the position of the Inner Tracker boxes and layers in the $\mathrm{x}-$ direction and to verify the correctness of the survey data. After applying corrections at the box and layer level the position of the $\mathrm{x}$-measuring ladders is known to a precision of $100 \mu \mathrm{m}$ or better [6]. The width of the residual distribution is given by the combination of the intrinsic detector resolution, misaligments and the effect of multiple scattering. The latter is not known since the particle momentum spectra is unknown. If the average momentum is around $5 \mathrm{GeV}$ the effect of multiple scattering would dominate.

The TT-Station consists of only four detection layers, so stand-alone tracking is not possible. However, alignment studies could be performed with the TED runs data using track candidates from the VELO extrapolated to the TT. The RMS of the hits associated to an extrapolated VELO track is about $500 \mu \mathrm{m}$ with $300 \mu \mathrm{m}$ expected from simulations for perfect alignment. The offset varies between 150-300 $\mu \mathrm{m}$ for the different layers of the TTStation, showing a quite precise alignment.
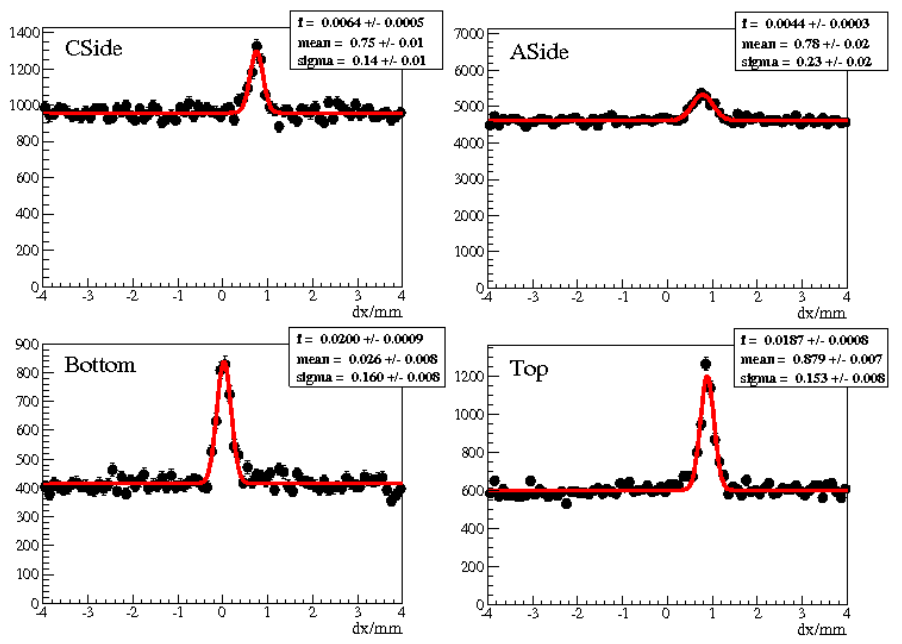

Figure 5: Residual distribution in station 2 for each box with TED events and using the survey data. The peaks correspond to the correct combination of hits, and the smallest peak is for the A-Side where the occupancy is highest. The offsets for the four boxes are obtained by fitting a Gaussian combined with a flat background.

The TED events have also been used for the internal relative time alignment of the different parts of the ST and for a first estimation of the signal-to-noise ratio $(\mathrm{S} / \mathrm{N})$. In Figure 6 reconstructed pulseshapes from runs taken with different sampling times of the Inner Tracker front end chip are shown. The peak time varies for the different parts of the detector due to differences in the cable lengths, time of flight of particles and the intrinsic delay of individual electronic elements. They all need to be fine tuned for choosing the optimal clock delays, which from the pulseshapes can be determined to an accuracy better than $1 \mathrm{~ns}$. This plot also gives the most probable value (MPV) of the charge measured in the sensor. Together with the noise measured for the detector modules this results in a detector $\mathrm{S} / \mathrm{N}$ of about 14 for the Inner Tracker and TT-Station modules, only $\sim 10 \%$ lower than expected [7].
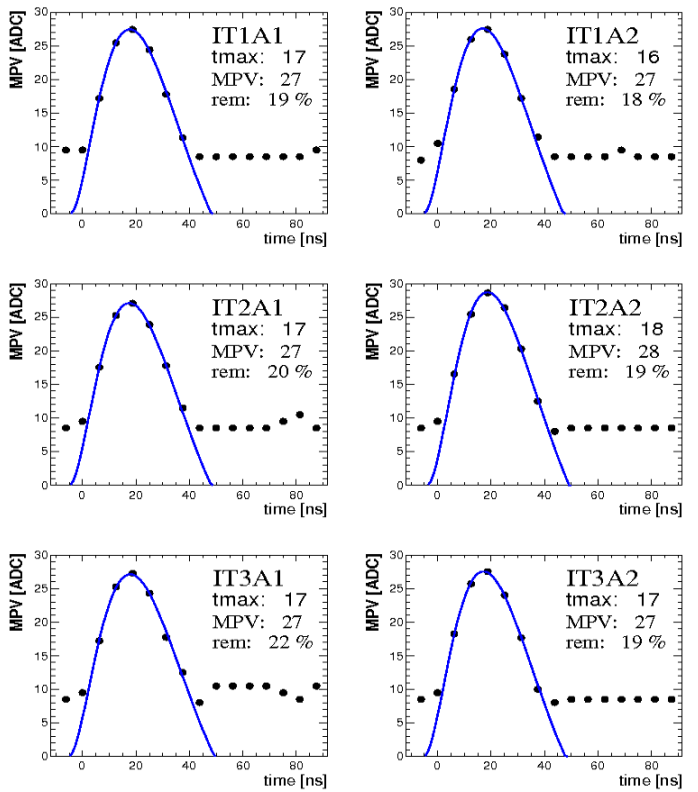

Figure 6: The most probable (MPV) of the sampled charge deposition in the silicon for different timing settings obtained in the TED run. IT1-IT3 refer to the three different Inner Tracker stations and A1, A2 to different regions within a station. The events were recorded during the TED runs. The fit is to the expected form for a $2^{\text {nd }}$ order CR-RC shaper.

\section{Summary}

The installation of the LHCb Silicon Tracker in the LHCb detector was completed in early summer 2008. Cosmic ray events as well as TED events from the LHC injection tests have been used for first alignment studies, the determination of the signal to noise ratio in the detector and also the internal time alignment. These preliminary studies showed that the relative alignment and signal to noise ratio are close to expectations.

\section{References}

[1] LHCb Reoptimized Detector Design and Performance Technical Design Report. CERN/LHCC-2003-030. ISBN 92-9083-209-6.

[2] The Beetle Reference Manual, N. Bakel et al., LHCb note 2001046.

[3] A. Vollhardt et al., Proc. LECC 2005, CERN/LHCC-2005-038 (2005) 187-191.

[4] O. Steinkamp, Nucl. Instr. and Meth. A 579 (2007) 736-741.

[5] Inner Tracker Survey Strategy, G. Conti et al., LHCb-2008-068; IT Survey measurements : Analysis and Implementaton in the LHCb Software, G.Conti, F.Blanc, LHCb-2008-069.

[6] First alignment of the Inner Tracker using data from the TI-8 sector test, M. Needham, LHCb-2009-030.

[7] Test-beam measurements on prototype ladders for the $\mathrm{LHCb}$ TT station and Inner Tracker, M. Agari et al., LHCb-2003-082; Layout and Expected Performance of the LHCb TT Station, J. Gassner,M. Needham, O. Steinkamp, LHCb-2003-140. 